\title{
PRactice of VENTilation in Patients with Novel Coronavirus Disease (PRoVENT-COVID): rationale and protocol for a national multicenter observational study in The Netherlands
}

\author{
Noor S. Boers ${ }^{1,2}$, Michela Botta ${ }^{1,2}$, Annisa M. Tsonas ${ }^{1,2}$, Anna Geke Algera ${ }^{1,2}$, Janesh Pillay ${ }^{3}$, \\ Dave A. Dongelmans ${ }^{1}$, Janneke Horn ${ }^{1}$, Alexander P. J. Vlaar ${ }^{1,2}$, Markus W. Hollmann ${ }^{2,4}$, \\ Lieuwe D. J. Bos ${ }^{1,2}$, Frederique Paulus ${ }^{1,2}$, Ary Serpa Neto ${ }^{5,6}$, Marcus J. Schultz ${ }^{1,2,7,8}$; \\ The PRoVENT-COVID investigators ${ }^{\dagger}$
}

\begin{abstract}
${ }^{1}$ Department of Intensive Care, ${ }^{2}$ Laboratory of Experimental Intensive Care and Anesthesiology (LEICA), Amsterdam UMC location AMC, The Netherlands; ${ }^{3}$ Department of Critical Care, University Medical Center Groningen, University of Groningen, The Netherlands; ${ }^{4}$ Department of Anaesthesiology, Amsterdam UMC location AMC, The Netherlands; ${ }^{5}$ Department of Critical Care Medicine, Hospital Isaelita Albert Einstein, Sao Paulo, Brazil; ${ }^{6}$ Australian and New Zealand Intensive Care Research Centre (ANZIC-RC), Monash University, Melbourne, Australia; ${ }^{7}$ Nuffield Department of Medicine, Oxford University, Oxford, UK; ${ }^{8}$ Mahidol-Oxford Tropical Medicien Research Unit (MORU), Mahidol University, Bangkok, Thailand

Contributions: (I) Conception and design: F Paulus, J Pillay, A Serpa Neto, MJ Schultz; (II) Administrative support: All authors; (III) Provision of study materials or patients: None; (IV) Collection and assembly of data: None; (V) Data analysis and interpretation: None; (VI) Manuscript writing: All authors; (VII) Final approval of manuscript: All authors.

Correspondence to: Dr. Janesh Pillay, MD, PhD. Department of Critical Care, University Medical Center Groningen, University of Groningen, Hanzeplein 1, 9713 GZ Groningen, The Netherlands. Email: j.pillay@umcg.nl.
\end{abstract}

\begin{abstract}
Background: The coronavirus disease 2019 (COVID-19) pandemic is rapidly expanding across the world, with more than 100,000 new cases each day as of end-June 2020. Healthcare workers are struggling to provide the best care for COVID-19 patients. Approaches for invasive ventilation vary widely between and within countries and new insights are acquired rapidly. We aim to investigate invasive ventilation practices and outcome in COVID-19 patients in the Netherlands.

Methods: PRoVENT-COVID ('study of PRactice of VENTilation in COVID-19') is an investigatorinitiated national, multicenter observational study to be undertaken in intensive care units (ICUs) in The Netherlands. Consecutive COVID-19 patients aged 18 years or older, who are receiving invasive ventilation in the participating ICUs, are to be enrolled during a 10-week period, with a daily follow-up of 7 days. The primary outcome is ventilatory management (including tidal volume expressed as $\mathrm{mL} / \mathrm{kg}$ predicted body weight and positive end-expiratory pressure expressed as $\mathrm{cmH}_{2} \mathrm{O}$ ) during the first 3 days of ventilation.
\end{abstract}

\footnotetext{
$\wedge$ ORCID: 0000-0001-8037-6638.

${ }^{\dagger}$ PRoVENT-COVID investigators: Jesse P. van Akkeren; Anna Geke Algera; Cheetel K. Algoe; Rombout B. van Amstel; Pablo van de Berg; Dennis C. J. J. Bergmans; Dido I. van den Bersselaar; Freke A. Bertens; Alexander J. G. H. Bindels; Jenni S. Breel; Caro L. Bruna; Milou M. de Boer; Sylvia den Boer; Noor S. Boers; Margriet Bogerd; Lieuwe D. J. Bos; Michela Botta; Onno L. Baur; Erik de Bruin; Laura A. Buiteman-Kruizinga; Olaf L. Cremer; Rogier M. Determann; Wim Dieperink; Jeanne van Dijk; Dave A. Dongelmans; Mart J. de Graaff; Michal S. Galek-Aldridge; Laura A. Hagens; Jasper J. Haringman; Sebastiaan T. van der Heide; Pim L. J. van der Heiden; Lotte L. Hoeijmakers; Liselotte Hol; Markus W. Hollmann; Janneke Horn; Robrecht van der Horst; Evy L. K. Ie; Dimitri Ivanov; Nicole P. Juffermans; Eline Kho; Eline S. de Klerk; Ankie W.M.M. Koopman-van Gemert; Matty Koopmans; Songul Kucukcelebi; Michael A. Kuiper; Dylan W. de Lange; Niels van Mourik; Sunny G. Nijbroek; Evelien A. N. Oostdijk; Frederique Paulus; Charlotte J. Pennartz; Janesh Pillay; Ilse M. Purmer; Thijs C.D. Rettig; Jan Paul Roozeman; Marcus J. Schultz; Ary Serpa Neto; Mengalvio E. Sleeswijk; Peter E. Spronk; Aart C. Strang; Willemke Stilma; Anissa M. Tsonas; Christel M. A. Valk; Alexander P. J. Vlaar; Lars I. Veldhuis; Ward H. van der Ven; Patricia van Velzen; Peter van Vliet; Peter H.J. van der Voort; Louis van Welie; Bas van Wijk; Tineke Winters; Wing Y. Wong; Arthur R. H. van Zanten.
} 
Secondary outcomes include other ventilatory variables, use of rescue therapies for refractory hypoxemia such as prone positioning and extracorporeal membrane oxygenation, use of sedatives, vasopressors and inotropes; daily cumulative fluid balances; acute kidney injury; ventilator-free days and alive at day 28 (VFD-28), duration of ICU and hospital stay, and ICU, hospital and 90-day mortality.

Discussion: PRoVENT-COVID will be the largest observational study to date, with high density ventilatory data and major outcomes. There is urgent need for a better understanding of ventilation practices, and the effects of ventilator settings on outcomes in COVID-19 patients. The results of PRoVENT-COVID will be rapidly disseminated through electronic presentations, such as webinars and electronic conferences, and publications in international peer-reviewed journals. Access to source data will be made available through local, regional and national anonymized datasets on request, and after agreement of the PRoVENT-COVID steering committee.

Trial Registration: PRoVENT-COVID is registered at clinicaltrials.gov (identifier NCT04346342).

Keywords: SARS-CoV-2; the coronavirus disease 2019 (COVID-19); critical care medicine; mechanical ventilation; ventilator-free days

Submitted Jul 02, 2020. Accepted for publication Aug 07, 2020.

doi: $10.21037 / \mathrm{atm}-20-5107$

View this article at: http://dx.doi.org/10.21037/atm-20-5107

\section{Introduction}

The coronavirus disease 2019 (COVID-19) is a lower respiratory tract infection caused by the highly contagious severe acute respiratory coronavirus-2 (SARS-CoV-2). The disease has rapidly spread across the globe, with over hundreds of thousands of new cases each day as of end-June 2020 (1). An estimated 5\% of hospitalized patients need admission to an intensive care unit (ICU) for respiratory support (2). Many of these patients require intubation for invasive ventilation, and in this group the case fatality rate is high (3).

While invasive ventilation can be life-saving in patients with acute respiratory distress syndrome (ARDS), it has a strong potential to worsen pre-existing lung injury or even initiate it (4). So-called ventilator-induced lung injury (VILI) is associated with increased mortality and a longer duration of ventilation in survivors (5). Lungprotective ventilation, mainly aiming at prevention of overdistension of open lung parts through use of a low tidal volume $\left(\mathrm{V}_{\mathrm{T}}\right)$ and low airway pressures, including plateau pressure (Pplat) (6) and driving pressure $(\Delta \mathrm{P})(7)$, reduces mortality, and is considered the standard of care (8-11). The benefit of positive end-expiratory pressure (PEEP) with or without recruitment maneuvers is less certain, and their use might actually cause harm to patients with ARDS (12). A randomized trial comparing low PEEP to 'personalized' high PEEP suggested benefit from high
PEEP in patients with 'recruitable' ARDS, but harm from high PEEP in patients with 'non-recruitable' ARDS (13). The findings from this trial have possible implications for patients with ARDS caused by COVID-19 (14).

Worldwide, in Europe and even in The Netherlands, opinions with regard to the way ventilators should be set in COVID-19 patients vary widely. For instance, we ourselves noticed remarkable differences in e.g., $\mathrm{V}_{\mathrm{T}}$ and $\triangle \mathrm{P}, \mathrm{PEEP}$, and use of prone positioning and neuromuscular blockade in patients transferred between hospitals in the Netherlands. However, whether these differences translate into differences in outcomes in COVID-19 patients, alike for what has been found in patients with ARDS, is uncertain. We aim to investigate invasive ventilation practices and outcomes in COVID-19 patients in The Netherlands. For this, PRoVENT-COVID (study of 'PRactice of VENTilation in COVID-19') is to be undertaken. This study will form an important first step in creating standard and guide development of guidelines for invasive ventilation in COVID-19 patients. Standardization and implementation of guidelines patients could improve outcome of COVID-19 patients.

\section{Methods}

\section{Design and setting}

PRoVENT-COVID is an investigator-initiated, 


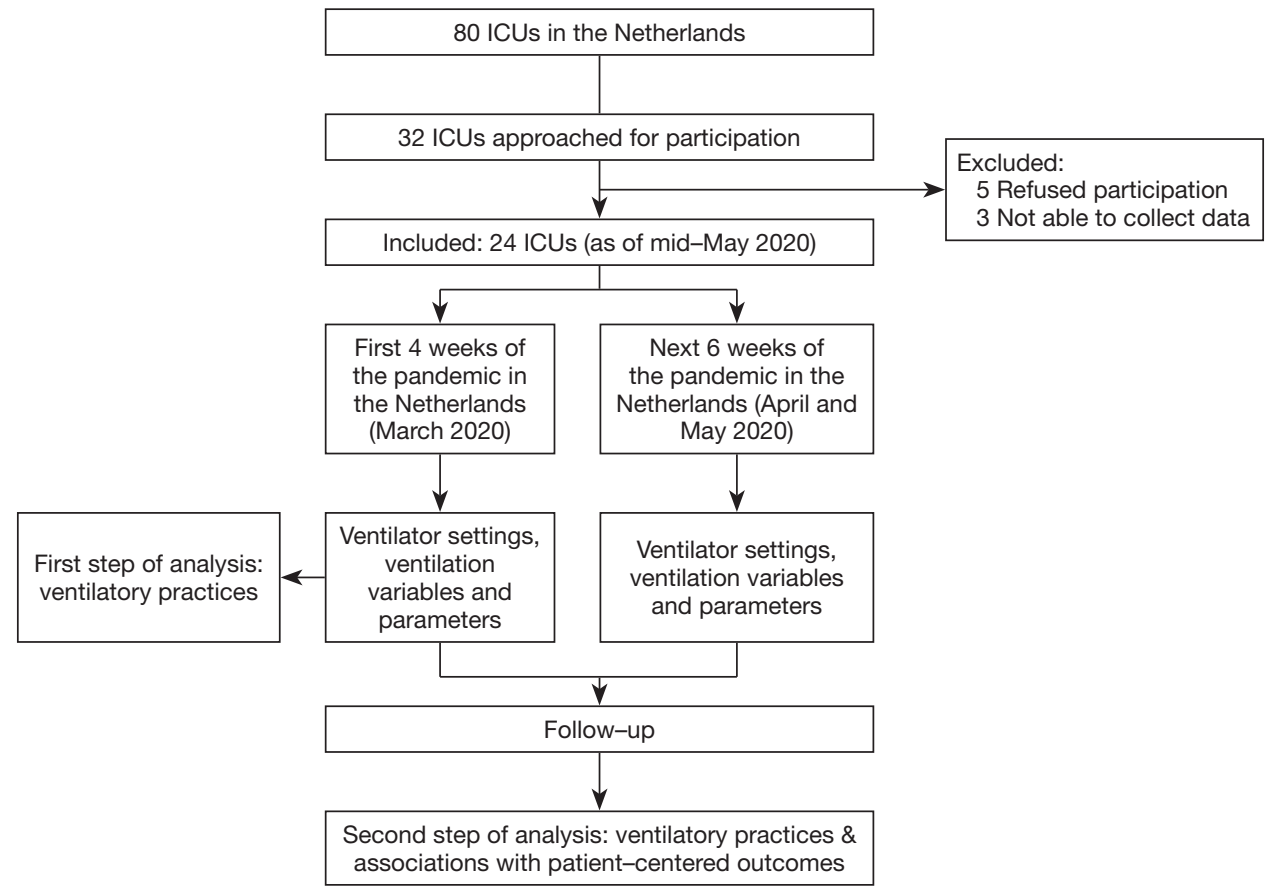

Figure 1 Flow chart of inclusion in PRoVENT-COVID. ICU, intensive care unit.

national, multicenter observational study in COVID-19 patients with respiratory failure for which they receive invasive ventilation in intensive care units (ICUs) in The Netherlands. PRoVENT-COVID will capture high density data in at least 24 ICUs. The study protocol is approved by the local Institutional Review Board of the Amsterdam University Medical Centers, location 'AMC' (W20_157 \# 20.171). PRoVENT-COVID is conducted in accordance with the Declaration of Helsinki and is registered at www.clinicaltrials.gov (trial identification number NCT04346342). Figure 1 shows the study flow chart.

\section{Study population}

At least 1,000 consecutively invasively ventilated COVID-19 patients admitted to participating ICUs are expected to be enrolled. Within each center, data capturing starts from the first COVID-19 patient admitted, and will continue enrolling patients for a total of 10 weeks. Since a substantial number of patients will be transported in between ICUs within The Netherlands, and to a lesser extend also to Germany, we will not be able to collect ventilation data of a subset of patients from start of ventilation (i.e., when invasive ventilation started in an ICU that does not participate in PRoVENT-COVID), or during the course of ventilation (i.e., when invasive ventilation will continue in an ICU that does not participate in PRoVENTCOVID). In all patients, however, duration of ventilation and mortality can and will be captured, even if transported to or from a non-participating ICU.

Inclusion criteria of PRoVENT-COVID are: COVID-19 infection confirmed by reverse transcriptase-polymerase chain reaction (RT-PCR), or highly suspected based on presence of typical abnormalities on chest computer tomography (CT) images (15). Apart from an age $<18$ years, this study has no exclusion criteria.

\section{Study conduct}

Local investigators screen patients with confirmed or suspected COVID-19 infection. Data collection has already started in some centers as of mid-May 2020 and will continue until the end of August 2020.

\section{Data to be collected}

Baseline and demographic variables will be collected from the first day of ventilation in each participating center. These variables include gender, age, weight and height, medication and comorbidities. In addition, date of hospital 
admission, date of ICU admission, date and exact timing of intubation and start of invasive ventilation (if possible, also in transferred patients), transferred with invasive ventilation (if applicable, duration of invasive ventilation in previous hospital (if applicable), use of non-invasive ventilation (NIV) before intubation (yes or no) and if so, duration (hours), Acute Physiology and Chronic Health (APACHE) II/IV score or Simplified Acute Physiology Score (SAPS) II, Sequential Organ Failure Scores (SOFA), and plasma creatinine in 24 hours before admission/intubation $(\mathrm{mmol} / \mathrm{L})$.

Within one hour of start of invasive ventilation, or within one hour of arrival in the ICU if a patient was transported under invasive ventilation from another ICU, and at 3 fixed time points per day (8:00 $\mathrm{AM}$, and 4:00 PM and 8:00 PM) up to day 3 , the following ventilation variables and parameters will be collected:

- Ventilation mode; inspiratory and expiratory tidal volume $\left(\mathrm{V}_{\mathrm{T}}\right)(\mathrm{mL})$; positive end-expiratory pressure (PEEP) $\left(\mathrm{cmH}_{2} \mathrm{O}\right)$; maximum airway pressure (Pmax) $\left(\mathrm{cmH}_{2} \mathrm{O}\right)$ or plateau pressure (Pplat) $\left(\mathrm{cmH}_{2} \mathrm{O}\right)$ or peak pressure (Ppeak) $\left(\mathrm{cmH}_{2} \mathrm{O}\right)$; level of pressure support above PEEP $\left(\mathrm{cmH}_{2} \mathrm{O}\right)$; inspired fraction of oxygen $\left(\mathrm{FiO}_{2}\right)(\%)$; set and measured respiratory rate (RR) $\left(\mathrm{min}^{-1}\right)$; inspiration to expiration ratio (I:E) (ratio); saturation of peripheral oxygen $\left(\mathrm{SpO}_{2}\right)(\%)$; and endtidal carbon dioxide $\left(\mathrm{etCO}_{2}\right)(\mathrm{kPa})$; and

- Arterial blood gas analysis (ABA) results including arterial $\mathrm{pH}$; partial pressure of oxygen $\left(\mathrm{PaO}_{2}\right)(\mathrm{kPa}$ or $\mathrm{mmHg})$; partial pressure of carbon dioxide $\left(\mathrm{PaCO}_{2}\right)$ $(\mathrm{kPa}$ or $\mathrm{mmHg})$; arterial bicarbonate $\left(\mathrm{HCO}_{3}-\right)$ $(\mathrm{mmol} / \mathrm{L})$; arterial saturation of oxygen $\left(\mathrm{SaO}_{2}\right)(\%)$; arterial lactate levels $(\mathrm{mmol} / \mathrm{L})$.

At the same timepoints as for ventilation data, hemodynamic parameters will be collected:

- Arterial blood pressure $(\mathrm{mmHg})$ and heart rate $\left(\mathrm{min}^{-1}\right)$.

In addition, the following outcome data will be collected daily at 08.00 in the first 3 days after admission:

- Life status (alive or deceased), location (in ICU, hospital or other facility), intubation status;

- Daily Sequential Organ Failure Assessment (SOFA) scores;

- Cumulative dose of sedatives (mg), cumulative dose of vasopressors $(\mathrm{mg})$ cumulative fluid balance during last 24 hours $(\mathrm{mL})$, amount of fluid administered during last 24 hours (mL), urine output (mL/hour);

- Plasma creatinine $(\mathrm{mmol} / \mathrm{L})$, the use of prone position (yes or no), and if yes, time and duration (hours);
- $A B G$ results in supine position, the use of recruitment manoeuvre (yes or no), use of veno-venous, venoarterial or arterio-venous extracorporeal membrane oxygenation (ECMO) (yes or no); and

- Occurrence of pneumothorax (yes or no).

Definitive follow-up data will be collected at days 7, 28 and 90:

- Life status at day 7, day 28 and day 90 (alive yes or no), and if not, the date of death:

- Location (ICU, hospital, home), intubated (yes or no), and if not, extubation date:

- Acute Kidney Injury (yes or no); and

- Date of discharge from ICU; and date of discharge from hospital.

Workflow of data collection is represented in Figure 2.

\section{Study endpoints}

The primary outcome is ventilatory management (including tidal volume, airway pressures, oxygen fraction and respiratory rate) during the first 3 days of ventilation. Secondary outcomes include other ventilation variables and parameters, including $\mathrm{ABA}$ results, use of rescue therapies (including prone positioning and ECMO), use of sedatives, vasopressors and inotropes, daily cumulative fluid balances, development of kidney injury, ventilator-free days and alive at day 28 (VFD-28), ICU, hospital and 90-day mortality, and duration of ICU and hospital stay in survivors.

\section{Definitions}

VFD-28 is defined as the number of days the patient has been completely liberated from the ventilator and alive during the first 28 days from the start of mechanical ventilation in a participating center. Duration of ICU and hospital stay is calculated from the first day of admission to a participating center and patient discharge (e.g., death, transfer to a non-participating center or discharge to home or chronic care facility) from the ICU or hospital, respectively. All-cause ICU or 90-day mortality is defined as any death occurring in the ICU or within 90 days of admission to a participating center, respectively.

\section{Data management}

Data will be collected from the locally used electronic patient data management systems. Either local investigators or investigators from the Amsterdam UMC, location 


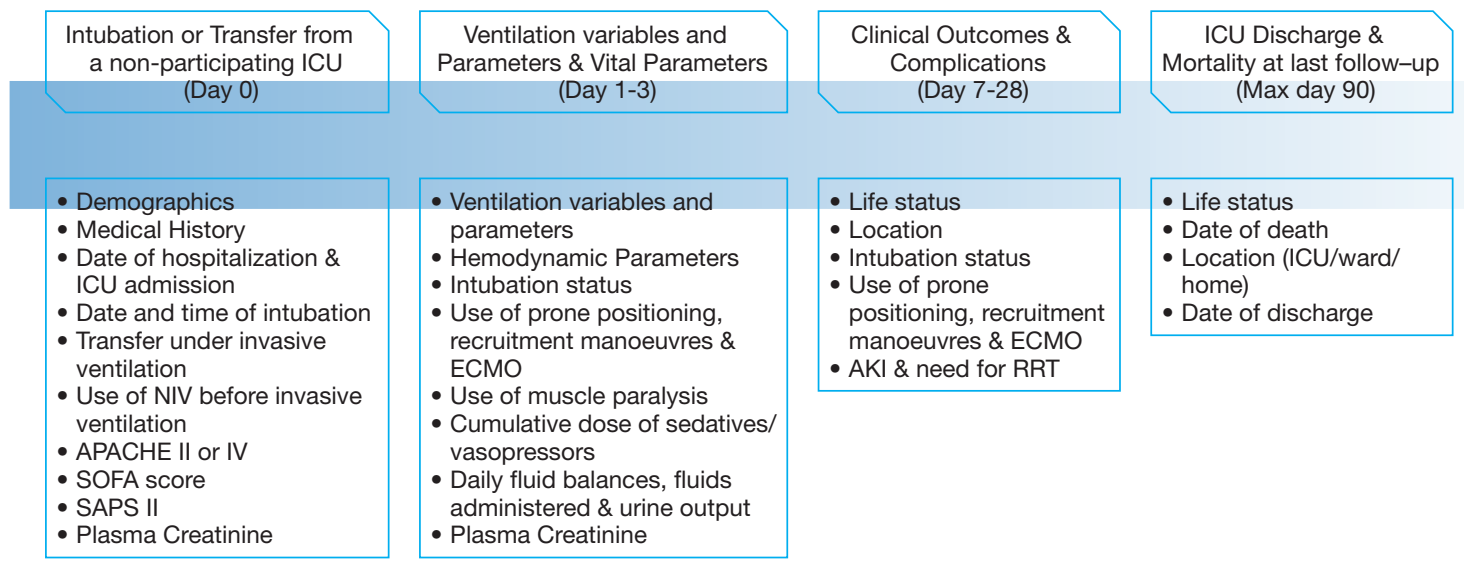

Figure 2 Sequence of data collection. ICU, intensive care unit; NIV, non-invasive ventilation; APACHE, Acute Physiology and Chronic Health Evaluation; SOFA score, Sequential Organ Failure Assessment score; SAPS II, Simplified Acute Physiology Score; ECMO, extracorporeal membrane oxygenation; AKI, acute kidney injury; RRT, renal replacement therapy.

AMC, will transcribe the collected data directly into an anonymized online electronic case report form (eCRF) (Castor Electronic Data Capture; https://castoredc. com). Access to the data-entry system is protected by a personalized username and password. A log with limited patient data will be completed of all included and excluded patients will be kept during the data collection period. This $\log$ will be deleted when data collection is complete.

\section{Study sites}

The following hospitals already agreed in participating in PRoVENT-COVID: Department of Intensive Care, University Medical Center Groningen, Groningen [1]; Medical Center Leeuwarden, Leeuwarden, The Netherlands [2]; Department of Intensive Care, ISALA hospital, Zwolle, The Netherlands [3]; Department of Intensive Care, Rijnstate hospital, Arnhem, The Netherlands [4]; Department of Intensive Care, Hospital group Twente, Almelo, The Netherlands [5]; Department of Intensive Care, Gelderse Vallei, Ede, The Netherlands [6]; Department of Intensive Care, Gelre hospitals, Apeldoorn [7]; Department of Intensive Care, University Medical Centers Utrecht, Utrecht, The Netherlands [8]; Department of Intensive Care, Antonius hospital, Nieuwegein, The Netherlands [9]; Department of Intensive Care, Amsterdam University medical centers [AMC], Amsterdam, The Netherlands [10]; Department of Intensive Care, Onze Lieve Vrouwe Gasthuis, Amsterdam, The Netherlands [11]; Department of Intensive Care,
Spaarne hospital, Hoofddorp, The Netherlands [12]; Department of Intensive Care, Dijklander hospital, Hoorn, The Netherlands [13]; Department of Intensive Care, Maasstad hospital, Rotterdam, The Netherlands [14]; Department of Intensive Care, HAGA hospital, Den Haag, The Netherlands [15]; Department of Intensive Care, Haaglanden Medical Centers, Den Haag, The Netherlands [16]; Department of Intensive Care, Reinier de Graaf hospital, Delft, The Netherlands [17]; St. Anna hospital, Geldrop [18]; Department of Intensive Care, Catharina hospital, Eindhoven, The Netherlands [19]; Department of Intensive Care, Amphia hospital, Breda, The Netherlands [20]; Department of Intensive Care, Maxima Medical Center, Eindhoven [21]; Department of Intensive Care, Zuyderland Hospital, Heerlen, The Netherlands [22]; Department of Intensive Care, Maastricht University Medical Center, Maastricht, The Netherlands [23]; Department of Intensive Care, Flevo Hospital, Almere, The Netherlands [24].

\section{Statistical analysis plan}

As the outcome of this study is urgently needed, data analysis will be done in two sequential steps. The first step includes an analysis of all patients admitted for invasive ventilation in one of the participating ICUs in the first four weeks after admission of the first COVID-19 patients, which was in all centers within the first weeks of March 2020. This part of the analysis focusses on ventilation practices in the first weeks of the pandemic in the 
Netherlands. This analysis may underestimate the effect of ventilation variables and parameters on outcomes, as many patients will not yet be weaned from the ventilator and remain admitted in the ICU. The second step includes an analysis of all COVID-19 patients admitted during the total 10 weeks PRoVENT COVID will enroll patients, with a complete follow up till day 90 . This analysis will provide an answer to the question whether certain ventilator settings, variables and parameters are associated with patientcentered and other important outcomes.

Descriptive statistics will be used to describe the study population, and data are expressed in number and relative proportions for categorical variables and median (quartile $25 \%$ - quartile $75 \%$ ) for continuous variables.

All analyses will be performed using multilevel (patients nested in hospitals), mixed modelling with hospitals as random effect. Ventilatory variables and parameters will be compared among the groups, and absolute differences with the respective $95 \%$-confidence interval (CI) will be calculated as the absolute difference from a mixed-effect linear model considering the hospitals as random effect to account for within-center clustering. Categorical variables will be compared as the risk difference from the same model.

Cumulative distribution plots will be used to demonstrate the cumulative distribution frequency of ventilation variables. Vertical dotted lines will represent the cut-off for each variable and the horizontal dotted lines the respective proportion of patients reaching each cut-off. Cut-offs to form matrices may use widely accepted values for each variable, specifically $8 \mathrm{~mL} / \mathrm{kg}$ PBW for tidal volume, $10 \mathrm{cmH}_{2} \mathrm{O}$ for PEEP, $30 \mathrm{cmH}_{2} \mathrm{O}$ for plateau pressure, and $15 \mathrm{cmH}_{2} \mathrm{O}$ for $\Delta \mathrm{P}$.

Mixed-effect multivariable logistic or linear regression model will used to identify factors independently associated with major outcomes, like death and VFD-28. Subanalyses are planned to investigate differences in ventilation practice and outcomes in the following prespecified subgroups: women versus men, and patients categorized by the body mass index.

All analyses will be conducted in R v.3.60 and a $\mathrm{P}$ value $<0.05$ will be considered statistically significant.

\section{Study organization}

The Steering Committee is composed of selected members of the PRoVENT-COVID investigator group. These investigators were involved in the design of this study and the analysis of the data. Data collection onsite and submission into the eCRF will performed either by external investigators from the Amsterdam UMC, location AMC, or local investigators. Incomplete or incorrectly entered eCRFs will be signalled to the collecting investigators by a central coordinator.

\section{Patient and public involvement}

Patients and public were not directly involved in any phase of this study.

\section{Ethics and dissemination}

This study will be conducted according to the principles of the Declaration of Helsinki (revision Fortaleza, Brazil, October 2013). Since the proposed study concerns a service review, no research related interventions will take place. Therefore, no ethical concerns exist. As pseudoanonymous data, which can no longer be attributed to a specific data subject will be used, there is no concern for informed consent. Participating centres will also submit the study protocol to their local institutional review board for judgment of feasibility. The results of this study will be published in a peer-reviewed medical journal. After publication of the primary results, access to source data will be made available by providing anonymized datasets on request and after agreement of the steering committee of PRoVENT-COVID.

\section{Discussion}

PRoVENT-COVID is designed and will be conducted to compare ventilation practice in COVID-19 patients in the Netherlands, and to determine whether differences in ventilator settings, variables and parameters have an independent association with important patientcentered outcomes. The findings of this service review may serve as a basis for highly-anticipated suggestions and recommendations in national and international guidelines for invasive ventilation in COVID-19 patients who need invasive ventilation because of respiratory failure.

Specific guidelines for invasive ventilation in COVID-19 are currently lacking, and practice is mainly based on evidence from studies performed in patients with ARDS due to another cause. PRoVENT-COVID will be the first study that combines epidemiology with ventilation practices and outcomes in invasively ventilated COVID-19 
patients. Other strengths of PRoVENT-COVID are as follows. First, this will be the largest study of ventilation practice and outcomes in invasively ventilated COVID-19 patients with high granularity in ventilator data. Second, the results of this service review will be available within weeks after completion of data-capturing in the first month of the pandemic, allowing fast optimization of ventilation practice in ICUs that care for COVID-19 patients. Next, wide representation of academic as well as non-academic hospitals, covering all provinces in the Netherlands, will allow a thorough national insight in ventilation practices and outcomes in critically ill COVID-19 patients.

This study has limitations. First, PRoVENT-COVID has a retrospective design. Therefore, conclusions drawn of associations between ventilation practices and outcomes should be interpreted with caution. In the surge of patients in need of invasive ventilation, this is an essential first step in early guidance of ventilatory practices. Another weakness of the study is that uncollected data might play a larger role then we anticipate. For instance, the COVID-19 pandemic has unleashed a myriad of small experimental trials which aim to limit pulmonary pathology or viral replication. Although to date no clear positive results have been published, between-center variation in these experimental practices could have an unknown influence on outcome data. In addition, the CRF used in this study was designed to increase feasibility of the study. Indeed, we decided to collect ventilation parameters only for the first 72 hours after start of ventilation, while it could be that ventilation practices beyond that timeframe also effect outcomes.

The results of PRoVENT-COVID are highly relevant for optimization of ventilation practice in critically ill COVID-19 patients. Swift generation and implementation of recommendations for invasive ventilation in these patients has the potential to improve outcomes. Furthermore, optimization of ventilation may lead to a reduced duration in of invasive ventilation, that may free up ICU capacity. As of this moment, ICU capacity is at the center of the public debate and seems to be a major determinant for societal and economic restrictions, therefore ultimately, reduction in intensive duration has the potential to influence these political decisions.

\section{Strengths and limitations of this study}

- The database of PRoVENT-COVID will contain high density data of invasive ventilation and relevant clinical outcomes in COVID-19 patients in the Netherlands;
- PRoVENT-COVID will have a large sample size which allows for a robust analysis of ventilation practices, and associations between ventilator settings and major outcomes;

- PRoVENT-COVID is limited by its observational nature and its national character; and

- The data generated by this service review will rapidly provide guidance to ventilation practice in COVID-19 patients in absence of randomized clinical trials of invasive ventilation

\section{Conclusions}

PRoVENT-COVID is designed to rapidly determine and compare ventilation practices, and to determine the impact of certain ventilation strategies on outcomes in COVID-19 patients receiving invasive ventilation in the Netherlands. The results of this study can help identifying best ventilation practices, and aid to generating recommendations for ventilation in COVID-19 patients.

\section{Acknowledgments}

Funding: None.

\section{Footnote}

Conflicts of Interest: All authors have completed the ICMJE uniform disclosure form (available at http://dx.doi. org/10.21037/atm-20-5107). The authors have no conflicts of interest to declare.

Ethical Statement: The authors are accountable for all aspects of the work in ensuring that questions related to the accuracy or integrity of any part of the work are appropriately investigated and resolved. This study will be conducted according to the principles of the Declaration of Helsinki (revision Fortaleza, Brazil, October 2013). Since the proposed study concerns a service review, no research related interventions will take place. Therefore, no ethical concerns exist. As pseudo-anonymous data, which can no longer be attributed to a specific data subject will be used, there is no concern for informed consent.

Open Access Statement: This is an Open Access article distributed in accordance with the Creative Commons Attribution-NonCommercial-NoDerivs 4.0 International License (CC BY-NC-ND 4.0), which permits the non- 


\section{Page 8 of 8}

commercial replication and distribution of the article with the strict proviso that no changes or edits are made and the original work is properly cited (including links to both the formal publication through the relevant DOI and the license). See: https://creativecommons.org/licenses/by-nc-nd/4.0/.

\section{References}

1. University JH. COVID-19 Dashboard by the Center for Systems Science and Engineering (CSSE) at Johns Hopkins University (JHU). 2020. Available online: https:// www.arcgis.com/apps/opsdashboard/index.html\#/bda7594 740fd40299423467b48e9ecf6.

2. Guan WJ, Ni ZY, Hu Y, et al. Clinical Characteristics of Coronavirus Disease 2019 in China. N Engl J Med 2020;382:1708-20.

3. Yang $\mathrm{X}, \mathrm{Yu} \mathrm{Y}, \mathrm{Xu} \mathrm{J}$, et al. Clinical course and outcomes of critically ill patients with SARS-CoV-2 pneumonia in Wuhan, China: a single-centered, retrospective, observational study. Lancet Respir Med 2020;8:475-81.

4. Slutsky AS, Ranieri VM. Ventilator-induced lung injury. N Engl J Med 2014;370:980.

5. Acute Respiratory Distress Syndrome N, Brower RG, Matthay MA, et al. Ventilation with lower tidal volumes as compared with traditional tidal volumes for acute lung injury and the acute respiratory distress syndrome. $\mathrm{N}$ Engl J Med 2000;342:1301-8.

6. Fan E, Del Sorbo L, Goligher EC, et al. An Official American Thoracic Society/European Society of Intensive Care Medicine/Society of Critical Care Medicine Clinical Practice Guideline: Mechanical Ventilation in Adult Patients with Acute Respiratory Distress Syndrome. Am J Respir Crit Care Med 2017;195:1253-63.

7. Amato MB, Meade MO, Slutsky AS, et al. Driving pressure and survival in the acute respiratory distress syndrome. $\mathrm{N}$

Cite this article as: Boers NS, Botta M, Tsonas AM, Algera AG, Pillay J, Dongelmans DA, Horn J, Vlaar APJ, Hollmann MW, Bos LDJ, Paulus F, Serpa Neto A, Schultz MJ; The PRoVENTCOVID investigators. PRactice of VENTilation in Patients with Novel Coronavirus Disease (PRoVENT-COVID): rationale and protocol for a national multicenter observational study in The Netherlands. Ann Transl Med 2020;8(19):1251. doi: 10.21037/atm-20-5107

Boers et al. PRactice of VENTilation in novel COronaVIrus Disease

Engl J Med 2015;372:747-55.

8. Esteban A, Ferguson ND, Meade MO, et al. Evolution of mechanical ventilation in response to clinical research. Am J Respir Crit Care Med 2008;177:170-7.

9. Esteban A, Frutos-Vivar F, Muriel A, et al. Evolution of mortality over time in patients receiving mechanical ventilation. Am J Respir Crit Care Med 2013;188:220-30.

10. Matthay MA, Ware LB, Zimmerman GA. The acute respiratory distress syndrome. J Clin Invest 2012;122:2731-40.

11. Needham DM, Colantuoni E, Mendez-Tellez PA, et al. Lung protective mechanical ventilation and two year survival in patients with acute lung injury: prospective cohort study. Bmj 2012;344:e2124.

12. Writing Group for the Alveolar Recruitment for Acute Respiratory Distress Syndrome Trial I, Cavalcanti AB, Suzumura EA, et al. Effect of Lung Recruitment and Titrated Positive End-Expiratory Pressure (PEEP) vs Low PEEP on Mortality in Patients With Acute Respiratory Distress Syndrome: A Randomized Clinical Trial. JAMA 2017;318:1335-45.

13. Constantin JM, Jabaudon M, Lefrant JY, et al. Personalised mechanical ventilation tailored to lung morphology versus low positive end-expiratory pressure for patients with acute respiratory distress syndrome in France (the LIVE study): a multicentre, single-blind, randomised controlled trial. Lancet Respir Med 2019;7:870-80.

14. Schultz MJ. High versus low PEEP in non-recruitable collapsed lung tissue: possible implications for patients with COVID-19. Lancet Respir Med 2020;8:e44.

15. Prokop M, van Everdingen W, van Rees Vellinga T, et al. CO-RADS - A categorical CT assessment scheme for patients with suspected COVID-19: definition and evaluation. Radiology 2020;296:E97-E104. 